\title{
A Model for Correlated Rician Fading Channels Based on a Finite Queue
}

\author{
Libo Zhong, Fady Alajaji, Senior Member, IEEE, and Glen Takahara, Member, IEEE
}

\begin{abstract}
We study the problem of approximating the family of hard-decision frequency-shift keying demodulated correlated flat Rician fading channels via a recently introduced queue-based channel (QBC) model for binary communication channels with memory. For a given "discretized" fading channel, we construct a QBC whose noise process is statistically "close" in the Kullback-Leibler sense to the error or noise process that is generated by the fading channel, and the modeling accuracy is evaluated in terms of noise autocorrelation function (ACF) and channel capacity. Numerical results indicate that the QBC provides a good approximation of the fading channels for a wide range of channel conditions. Furthermore, it estimates the noise ACF more accurately than the finite-state Markov models that have been recently studied by Pimentel $e t$ al., while, at the same time, remaining mathematically tractable.
\end{abstract}

Index Terms-Autocorrelation function (ACF), capacity, correlated Rician fading, error statistics, Kullback-Leibler divergence rate (KLDR), modeling of communication channels with memory.

\section{INTRODUCTION}

I N RECENT years, there has been an increasing interest in transmitting voice, data, image, and video signals over wireless communication channels. However, wireless channels undergo a variety of time-varying signal impairments caused by propagation loss, shadowing, multipath fading, and thermal noise. In particular, it is important to understand the deleterious effects of fading on wireless transmission. A common feature of many fading channels is that they cause symbol errors to occur in clusters or bursts [2].

In the presence of error bursts, interleaving is usually applied to destroy or mitigate the memory because most coding systems and protocols are designed under the assumption of memoryless error processes. With perfect or ideal interleaving, it is possible to model the fading channels as memoryless channels. However, the use of interleaving introduces extra delay and complexity, and perfect interleavers do not exist in any practical system. In real-time personal communication systems, data are transmitted in short blocks, and fairly strict delay constraints must be obeyed (e.g., see [3]). Noninterleaved

Manuscript received July 4, 2005; revised July 24, 2006 and January 10, 2007. This work was supported in part by the Natural Sciences and Engineering Research Council of Canada and the Premier Research Excellence Award of Ontario. Parts of this work were presented at the IEEE 62nd Semiannual Vehicular Technology Conference, Dallas, TX, September 2005 [1]. The review of this paper was coordinated by Prof. H. Leib.

The authors are with the Department of Mathematics and Statistics, Queen's University, Kingston, ON K7L 3N6, Canada (e-mail: takahara@mast. queensu.ca).

Digital Object Identifier 10.1109/TVT.2007.904502 or finite-interleaved packet transmission over fading channels has recently received significant attention [4], [5].

Therefore, in this paper, we start with the premise that the inherent memory of fading communication channels cannot be neglected. An advantageous feature of memory is that the channel quality in the near future can be forecast based on the knowledge of previous channel conditions due to the statistical dependence of errors. To obtain highly reliable data transmission over channels with memory, we should take advantage of the channel memory by constructing effective error control coding strategies. For this reason, it is critical to fully understand the error structure of such channels. This is achieved via channel modeling, where the main objective is to provide a model whose properties are complex enough to closely capture the real channel statistical characteristics and simple enough to allow mathematically tractable system analysis. In this paper, we employ a binary additive channel model with memory that is based on a finite queue that reliably and tractably describes a family of correlated fading channels.

During the past several decades, a variety of channel models have been proposed and studied for the modeling of wireless fading channels (e.g., see [6]-[8] and the models therein). A finite-state Markov channel (FSMC) is a discrete valued channel with a finite set of possible states whose transition is governed by an underlying Markov chain and with a probability assignment that is independent of time [2], [9], [10]. FSMCs have been widely adopted for the description of the correlation structures and success/failure processes of wireless channels with bursty behavior [11] because they are efficient in quick simulations, system performance evaluations, and protocol investigations. Two of the earliest FSMC models for representing the "discretized" version (under hard-decision demodulation) of binary-input fading channels with memory are the Gilbert-Elliott channel (GEC) [12], [13] and the Fritchman channel [14]. They were, for example, employed to model highfrequency channels [15], mobile radio channels [16]-[18], low earth orbit satellite channels [19], and magnetic tape recorders [20]. The GEC model has been also used to evaluate the performance of coding and decoding schemes over bursty channels [3], [21], [22].

Many FSMC models including the aforementioned works have been proposed to fit realistic wireless channels. Wang and Moayeri [23] proposed an FSMC based on the partitioning of the received SNR into a finite number of states to model Rayleigh fading channels. The same approach was also independently presented in [4] and used in [24] and [25]. The model proposed in [23] attracted much attention because it has a good balance between accuracy and complexity. It was applied to 
the evaluation of system-related channel properties (such as the correlation properties of the fading mobile radio channel) in [26] and [27] by modeling the channel as a first-order Markov process whose transition probabilities are a function of the channel characteristics. In [28], an analytical model was used to evaluate the effect of mobile velocity on the performance of a communication system operating in a multipath fading channel.

FSMCs are often generated via finite-state hidden Markov models (HMMs). ${ }^{1}$ General HMMs were studied in [29] to model flat-fading channels. Due to their HMM structure, such channels can be difficult to mathematically analyze (e.g., they do not admit an exact closed-form expression for their capacity, and/or their block transition distribution is not transparently expressed in terms of the channel parameters), particularly when incorporated within an overall source and/or channel coded system. This may partly explain why, to date, few coding techniques that effectively exploit the noise memory have been successfully constructed for HMM-based channel models and for channels with memory in general. Instead, most current wireless communication systems are designed for memoryless channels and employ channel interleaving in an attempt to disperse the channel memory and make the channel appear memoryless (even burst-error correcting codes such as Reed-Solomon codes operating on an HMM-based channel perform best when interleaving is used, e.g., see [5]). However, in addition to the increased complexity/delay caused by interleaving, the failure to exploit the channel's memory at the encoder and/or decoder leads to a waste of channel capacity since it is well known that memory increases capacity ${ }^{2}$ for a wide class of channels (the class of information stable channels [31], [32]). It is, therefore, vital to construct channel models that can well represent the behavior of real-world channels while remaining analytically tractable for design purposes.

Alajaji and Fuja [32, Sec. VI] proposed a simple binary additive noise channel with memory, which is referred to as the finite memory contagion channel (FMCC), where the noise process is generated via a finite-memory version of Polya's urn scheme for the spread of a contagious disease through a population [33]. In such a channel, every error (or "infection," if we use the contagion interpretation) effectively increases the probability of future errors [33] and, hence, may lead to a clustering or burst of errors (i.e., an "epidemic" in the population). The resulting channel, which is fully described by only three parameters, has a stationary ergodic $M$ th-order Markov noise source and admits single-letter analytical expressions for its block transition distribution and capacity. This model was adopted in several source-channel and channel coding studies (e.g., [34]-[42]), where the channel statistics are incorporated into the system design to exploit the noise memory.

The queue-based channel (QBC), which was recently introduced in [43]-[45], is a binary additive noise channel with memory based on a finite queue. The QBC is a more general

\footnotetext{
${ }^{1} \mathrm{~A}$ description of a large class of finite- or infinite-state HMM-based channel models is provided in [2].

${ }^{2}$ In other words, the capacity of the "equivalent" memoryless channel that is achieved by ideal interleaving (with infinite interleaving span) is smaller than the capacity of the original channel (e.g., see [30]).
}

model than the FMCC as it subsumes the latter as a special case. It also features a stationary ergodic $M$ th-order Markov noise source, and it is fully characterized by four parameters $(\epsilon, \alpha, p$, and $M)$, thus having one more degree of freedom than the FMCC (by setting $\alpha=1$, the QBC reduces to the FMCC for the same bit error rate (BER), correlation coefficient, and memory order). It is important to point out that Pimentel et al. [46] have recently shown in a numerical study that the class of binary channel models with additive $K$ th-order Markov noise (to which the QBC belongs) is a good approximation, in terms of the autocorrelation function (ACF) and variational distance, to the family of hard-decision frequency-shift keying (FSK) demodulated time-correlated flat Rayleigh and Rician fading channels for a good range of fading environments, particularly for medium and fast fading rates. Note, however, that the general $K$ th-order Markov noise channels that were considered in [46] have a complexity (number of parameters) that grows exponentially with $K$, rendering it impractical for the modeling of channels with large memory, such as very slow Rayleigh fading channels (e.g., see Fig. 2 or [46, Fig. 11]). The QBC model, on the other hand, does not suffer from this limitation, as it has a fixed number of parameters (four parameters), and it can accommodate very large values of memory $M$. Like the FMCC, it enjoys a transparent formula for its $n$-fold statistics and a closed-form formula for its capacity, which are appealing features as they provide powerful analytical tools for code design and system analysis. In a recent related work [47], the problem of modeling the GEC using the QBC has been investigated, and it was shown (numerically) that the QBC provides a good approximation of the GEC for various channel conditions.

In this paper, we investigate the problem of approximating the same class of Rician fading channels studied in [46] via the QBC. Specifically, for a given hard-decision demodulated fading channel, we construct a QBC whose error (i.e., noise) process is statistically as close as possible to the error process generated by the fading channel. This is achieved by selecting the QBC parameters that minimize the Kullback-Leibler divergence rate (KLDR) between both noise processes for identical BERs and correlation coefficients. Since the QBC model has a simple Markovian structure and low complexity as it is fully described by only four parameters (while still allowing for large memory values), the optimization problem involves only two parameters and can be efficiently numerically solved. Modeling results indicate that the QBC is a good fit for fading channels as it accurately models (in terms of ACF and capacity) their bursterror behavior for a wide range of channel environments, including slow fading. The QBC is, thus, an interesting alternative to existing models for channels with memory (such as HMMs), which tend to be either too complex for tractable analysis and code design that exploits the channel's memory or too limiting for realistic modeling.

The rest of this paper is organized as follows. Preliminaries on the GEC and QBC channel models are presented in Section II. In Section III, we investigate the modeling of the Rician fading channels via the QBC. In Section IV, we provide the numerical fitting results. For the sake of comparison, we also model the fading channels via the GEC (which has the 
same number of parameters as the QBC) using the parameterization method of Pimentel et al. [46]. The accuracy of both methods is evaluated in terms of ACF and capacity. In Section V, we conclude with a summary along with several directions for future work.

\section{GEC AND QBC BINARY ChANNEL MODELS}

Hereafter, a discrete-time binary additive noise communication channel refers to a channel with common input, noise, and output alphabet $\mathcal{X}=\mathcal{Z}=\mathcal{Y}=\{0,1\}$, described by $Y_{n}=$ $X_{n} \oplus Z_{n}$, for $n=1,2,3, \ldots$, where $\oplus$ denotes addition modulo 2, and where $X_{n}, Z_{n}$, and $Y_{n}$, respectively, denote the channel's input, noise, and output at time $n$. Hence, a transmission error occurs at time $n$ whenever $Z_{n}=1$. It is assumed that the input and noise sequences are independent of each other. In this paper, a given noise process $\left\{Z_{n}\right\}_{n=1}^{\infty}$ will be generated according to one of the GEC, the QBC, and the discretized Rician fading channel.

\section{A. GEC}

The GEC model [12], [13], [30] is driven by an underlying stationary ergodic Markov chain $\left\{S_{k}\right\}$ with two states: a good state and a bad state, denoted by $G$ (or state 0 ) and $B$ (or state 1), respectively. In a fixed state, the channel behaves like a binary symmetric channel (BSC). The GEC is, thus, a timevarying $\mathrm{BSC}$, where $p_{G}$ and $p_{B}$ are the crossover probabilities in the good and bad states, respectively (the Gilbert channel [12] is obtained when $p_{G}=0$, i.e., it behaves like a noiseless BSC in the good state). After every channel transmission, the chain makes a state transition according to the following transition probability matrix:

$$
\begin{aligned}
\boldsymbol{P} & \triangleq\left[\begin{array}{cc}
\operatorname{Pr}\left\{S_{k}=0 \mid S_{k-1}=0\right\} & \operatorname{Pr}\left\{S_{k}=1 \mid S_{k-1}=0\right\} \\
\operatorname{Pr}\left\{S_{k}=0 \mid S_{k-1}=1\right\} & \operatorname{Pr}\left\{S_{k}=1 \mid S_{k-1}=1\right\}
\end{array}\right] \\
& =\left[\begin{array}{cc}
1-b & b \\
g & 1-g
\end{array}\right]
\end{aligned}
$$

where $0<b<1$, and $0<g<1$. A useful approach for calculating the probability of an error or noise sequence for the GEC is discussed in [6]. The probability of a noise sequence of length $n, z^{n}=\left(z_{1}, z_{2}, \ldots, z_{n}\right)$ can be expressed as

$$
P_{\mathrm{GEC}}\left(z^{n}\right) \triangleq P_{\mathrm{GEC}}\left\{Z^{n}=z^{n}\right\}=\boldsymbol{\pi}^{T}\left(\prod_{k=1}^{n} \boldsymbol{P}\left(z_{k}\right)\right) \mathbf{1}
$$

where ${ }^{T}$ denotes transposition, $P\left(z_{k}\right)$ is a $2 \times 2$ matrix whose $(i, j)$ th entry is the probability that the output symbol is $z_{k}$ when the chain makes a transition from state $S_{k-1}=i$ to $S_{k}=j$, i.e.,

$$
\begin{aligned}
& P(0)=\left[\begin{array}{cc}
(1-b)\left(1-p_{G}\right) & b\left(1-p_{B}\right) \\
g\left(1-p_{G}\right) & (1-g)\left(1-p_{B}\right)
\end{array}\right] \\
& P(1)=\left[\begin{array}{cc}
(1-b) p_{G} & b p_{B} \\
g p_{G} & (1-g) p_{B}
\end{array}\right]
\end{aligned}
$$

1 is the 2-D vector with all ones, and the vector $\pi$ indicates the stationary distribution vector of the underlying Markov chain, i.e.,

$$
\boldsymbol{\pi}=\left[\begin{array}{l}
\pi_{0} \triangleq \operatorname{Pr}\left\{S_{k}=0\right\} \\
\pi_{1} \triangleq \operatorname{Pr}\left\{S_{k}=1\right\}
\end{array}\right]=\left[\begin{array}{c}
\frac{g}{b+g} \\
\frac{b}{b+g}
\end{array}\right] .
$$

\section{B. QBC With Memory}

The additive noise process of the queue-based binary channel with memory [43]-[45] is generated according to a sampling mechanism involving the following two parcels.

- Parcel 1 is a queue of length $M$ that initially contains $M$ balls: either red or black.

- Parcel 2 is an urn that contains a very large number of balls, where the proportion of black balls is $1-p$, and the proportion of red balls is $p$, where $p \in(0,1), p \ll 1 / 2$.

We assume that the probability of selecting parcel 1 (the queue) is $\varepsilon$, whereas the probability of selecting parcel 2 (the urn) is $1-\varepsilon$, and $\varepsilon \in[0,1)$. Notice that the channel is actually a BSC with crossover probability $p$ when $\varepsilon=0$; in this case, we experiment on the urn only.

The noise process $\left\{Z_{n}\right\}_{n=1}^{\infty}$ is generated according to the following procedure. By flipping a biased coin [with $\operatorname{Pr}($ Head $)=\varepsilon$ ], we select one of the two parcels (select the queue if Heads and the urn if Tails). If parcel 2 (the urn) is selected, a pointer randomly points at a ball and identifies its color. If parcel 1 (the queue) is selected, the procedure is determined by the length of the queue. If $M \geq 2$, a pointer points at the ball in cell $k$ with probability $1 /(M-1+\alpha)$, for $k=1,2, \ldots, M-1$ and $\alpha \geq 0$, and points at the ball in cell $M$ with probability $\alpha /(M-1+\alpha)$ and identifies its color. If $M=1$, a pointer points at the ball in the only cell of the queue with probability 1 ; in this case, we set $\alpha=1$. If the selected ball from either parcel is red (black), we introduce a red (black) ball in cell 1 of the queue, pushing the last ball in cell $M$ out. The noise process $\left\{Z_{n}\right\}_{n=1}^{\infty}$ is then modeled as follows:

$$
Z_{n}= \begin{cases}1, & \text { if the } n \text {th experiment points at a red ball } \\ 0, & \text { if the } n \text {th experiment points at a black ball. }\end{cases}
$$

It can be shown that the resulting channel noise process $\left\{Z_{n}\right\}_{n=1}^{\infty}$ is a stationary ergodic (irreducible) $M$ th-order Markov process. Moreover, various statistical and information theoretic quantities of the $\mathrm{QBC}$, such as the channel block transition probability, capacity, and $\mathrm{ACF}$, can be determined (in closed form) in terms of $M, p, \varepsilon$, and $\alpha$ [43]-[45]. The expressions for these quantities are herein summarized.

Block Transition Probability: For a given input block $X^{n}=$ $\left(X_{1}, \ldots, X_{n}\right)$ and a given output block $Y^{n}=\left(Y_{1}, \ldots, Y_{n}\right)$, where $n$ is the block length, the channel block transition probability is

$$
P_{\mathrm{QBC}}^{(M)}\left\{Y^{n}=y^{n} \mid X^{n}=x^{n}\right\}=P_{\mathrm{QBC}}^{(M)}\left\{Z^{n}=z^{n}\right\} \triangleq P_{\mathrm{QBC}}^{(M)}\left(z^{n}\right)
$$


where $z_{i}=x_{i} \oplus y_{i}$, for $i=1,2, \ldots, n$, and the noise $n$-fold distribution is as follows.

- For block length $n \leq M$, the distribution is given by (4), shown at the bottom of the page, where $d_{a}^{b}=z_{b}+z_{b-1}+$ $\cdots+z_{a}\left(d_{a}^{b}=0\right.$ if $\left.a>b\right)$, and $\prod_{j=0}^{a}(\cdot) \triangleq 1$ if $a<0$.

- For block length $n \geq M+1$

$$
\begin{aligned}
P_{\mathrm{QBC}}^{(M)}\left(z^{n}\right)= & L^{(M)} \prod_{i=M+1}^{n}\left[\left(d_{i-M+1}^{i-1}+\alpha z_{i-M}\right)\right. \\
& \left.\times \frac{\varepsilon}{M-1+\alpha}+(1-\varepsilon) p\right]^{z_{i}} \\
\times & \left\{\left[\left(M-1-d_{i-M+1}^{i-1}\right)+\alpha\left(1-z_{i-M}\right)\right]\right. \\
& \left.\times \frac{\varepsilon}{M-1+\alpha}+(1-\varepsilon)(1-p)\right\}^{1-z^{i}}
\end{aligned}
$$

where $L^{(M)}$ is as shown at the bottom of the page.

Note that the channel's BER and noise correlation coefficient are given by the following:

$$
\mathrm{BER}_{\mathrm{QBC}}=P_{\mathrm{QBC}}^{(M)}\left\{Z_{i}=1\right\}=P_{\mathrm{QBC}}^{(M)}\left\{Z_{1}=1\right\}=p
$$

and

$$
\operatorname{Cor}_{\mathrm{QBC}}=\frac{E\left[Z_{2} Z_{1}\right]-E\left[Z_{2}\right] E\left[Z_{1}\right]}{\operatorname{Var}\left[Z_{1}\right]}=\frac{\frac{\varepsilon}{M-1+\alpha}}{1-\frac{M-2+\alpha}{M-1+\alpha} \varepsilon}
$$

respectively, where $E[\cdot]$ denotes expectation, and $\operatorname{Var}[\cdot]$ is the variance.

$A C F$ : The ACF of a binary stationary process $\left\{Z_{n}\right\}_{n=1}^{\infty}$ is defined by $R[m]=E\left[Z_{i} Z_{i+m}\right]=P_{\mathrm{QBC}}^{(M)}\left\{Z_{i}=1, Z_{i+m}=1\right\}$. It can be shown that the ACF of the QBC satisfies (8), shown at the bottom of the page.

Capacity: Since the QBC is a channel with stationary ergodic additive noise, it is information stable, and its (opera- tional) capacity $C_{\mathrm{QBC}}^{(M)}$ is given by the following familiar mutual information rate formula (e.g., [48]):

$$
C_{\mathrm{QBC}}^{(M)}=\lim _{n \rightarrow \infty} \sup _{X^{n}} \frac{1}{n} I\left(X^{n} ; Y^{n}\right)
$$

where $I(\cdot ; \cdot)$ denotes mutual information [49]. It can be shown that input $n$-tuples $X^{n}$ that are uniformly distributed over $\{0,1\}^{n}$ maximize $I\left(X^{n} ; Y^{n}\right)$; this yields the following expression for $C_{\mathrm{QBC}}^{(M)}$ :

$$
\begin{aligned}
C_{\mathrm{QBC}}^{(M)}= & 1-\sum_{\omega=0}^{M-1}\left(\begin{array}{c}
M-1 \\
\omega
\end{array}\right) L_{\omega}^{(M)} \\
& \times h_{b}\left[\omega \frac{\varepsilon}{M-1+\alpha}+(1-\varepsilon) p\right] \\
& -\sum_{\omega=1}^{M}\left(\begin{array}{c}
M-1 \\
\omega-1
\end{array}\right) L_{\omega}^{(M)} \\
& \times h_{b}\left[(\omega+\alpha-1) \frac{\varepsilon}{M-1+\alpha}+(1-\varepsilon) p\right]
\end{aligned}
$$

where $L_{\omega}^{(M)}$ is as shown at the bottom of the next page, $h_{b}(x) \triangleq-x \log _{2} x-(1-x) \log _{2}(1-x)$ is the binary entropy function, and $\prod_{j=0}^{a}(\cdot) \triangleq 1$ for $a<0$. Finally, it should be noted that the FMCC channel of [32] is a special case of the QBC: By setting $\alpha=1$, the $\mathrm{QBC}$ reduces to the FMCC (for identical $\mathrm{BER}$, Cor, and memory). Hence, the QBC is a more flexible channel model than the FMCC due to an additional degree of freedom.

\section{Fitting Rician Fading Channels Via the QBC}

We next consider the problem of fitting discretized Rayleigh and Rician fading channels via the QBC model. For the sake of comparison, we also model the fading channels via the

$$
P_{\mathrm{QBC}}^{(M)}\left(z^{n}\right)=\frac{\prod_{j=0}^{n-d_{1}^{n}-1}\left[j \frac{\varepsilon}{M-1+\alpha}+(1-\varepsilon)(1-p)\right] \prod_{j=0}^{d_{1}^{n}-1}\left[j \frac{\varepsilon}{M-1+\alpha}+(1-\varepsilon) p\right]}{\prod_{j=M-n}^{M-1}\left[1-(\alpha+j) \frac{\varepsilon}{M-1+\alpha}\right]}
$$

$$
L^{(M)}=\frac{\prod_{j=0}^{M-1-d_{1}^{M}}\left[j \frac{\varepsilon}{M-1+\alpha}+(1-\varepsilon)(1-p)\right] \prod_{j=0}^{d_{1}^{M}-1}\left[j \frac{\varepsilon}{M-1+\alpha}+(1-\varepsilon) p\right]}{\prod_{j=0}^{M-1}\left[1-(\alpha+j) \frac{\varepsilon}{M-1+\alpha}\right]}
$$

$$
R_{\mathrm{QBC}}[m]= \begin{cases}\frac{p}{\frac{1+\alpha}{M-1+\alpha}+(1-\varepsilon) p} p, & \text { if } m=0 ; \\ \left(1-\frac{M-1+\alpha}{M-\alpha}\right) & \text { if } 1 \leq m \leq M-1 ; \\ \left(1-\frac{\varepsilon}{2}+\frac{\varepsilon}{M-1+\alpha}\left(\sum_{i=m-M+1}^{m-1} R_{\mathrm{QBC}}[i]+\alpha R_{\mathrm{QBC}}[m-M]\right),\right. & \text { if } m \geq M\end{cases}
$$




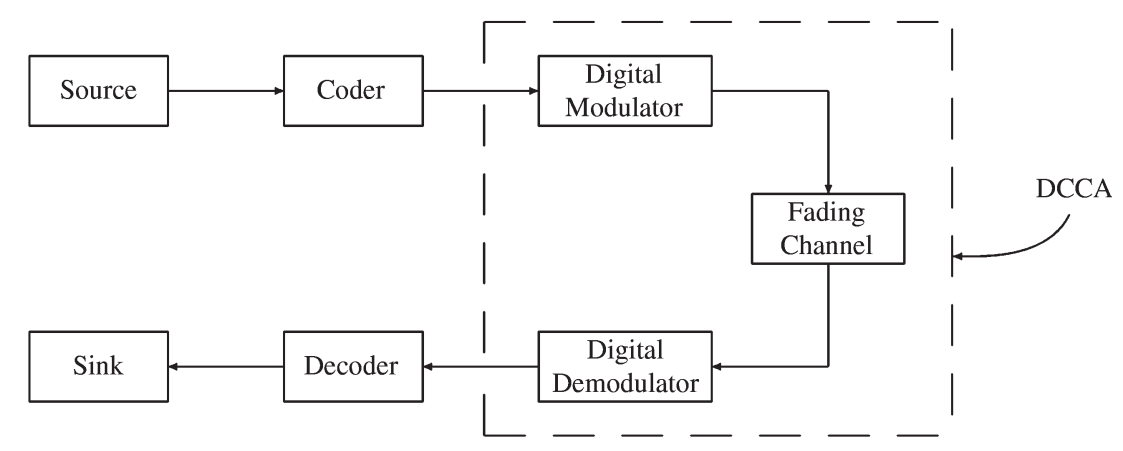

Fig. 1. Overall system and the equivalent DCCA model.

GEC (which has the same number of parameters as the QBC) according to the parameterization method of Pimentel et al. [46]. The accuracy of both methods is evaluated in terms of ACF and capacity.

\section{A. Fading Channel Model}

We consider a discrete (binary input, binary output) communication system, referred to as the discrete channel with Clarke's autocorrelation (DCCA) model, that employs binary FSK modulation, a time-correlated Rician flat-fading channel, and a hard quantized noncoherent demodulation [46]. As in [6], [46], and [50], we consider noncoherent FSK modulation; however, any other modulation/demodulation scheme for which the expression of the probability of length $n$ error sequences is available [as in (11) below] can be also studied by our model. The complex envelope of the received signal at the input to the demodulator is corrupted by a multiplicative Rician fading and by an additive white Gaussian noise, i.e.,

$$
\tilde{R}(t)=\sqrt{2 E_{s}} \tilde{G}(t) \tilde{S}(t)+\tilde{N}(t)
$$

where $E_{s}$ is the symbol energy. $\tilde{S}(t)$ is the complex envelope of the symbol, which can be expressed as $\tilde{S}(t)=\sum_{k=1}^{\infty} p_{a_{k}}(t-$ $k T)$, where the binary information bearing symbols $a_{k}$ are embedded in the signals $p_{i}(t), i=0,1$, which are equally probable orthogonal signals with unit energy. $T$ is the symbol interval, and $\tilde{N}(t)$ is the complex envelope of the white Gaussian noise with ACF given by $(1 / 2) E\left[\tilde{N}(t+\tau) \tilde{N}^{*}(t)\right]=$ $N_{0} \delta(\tau)$, where $N_{0}$ is the variance of $N(t)$ [7]. The complex envelope of the fading process $\tilde{G}(t)=\tilde{G}_{I}(t)+j \tilde{G}_{Q}(t)$ is a complex wide sense stationary Gaussian process with mean $\eta$, $j=\sqrt{-1}$, and the quadrature components $\tilde{G}_{I}(t)$ and $\tilde{G}_{Q}(t)$ are mutually independent Gaussian processes with the same covariance function $\operatorname{Cov}(\tau)$, which, adopting Clarke's fading model [51], [52], can be expressed as

$$
\begin{aligned}
\operatorname{Cov}(\tau) & =\frac{1}{2} E\left[(\tilde{G}(t+\tau)-\eta)\left(\tilde{G}^{*}(t)-\eta\right)\right] \\
& =\sigma_{g}^{2} J_{0}\left(2 \pi f_{D} \tau\right)
\end{aligned}
$$

where

$$
J_{0}(x)=\sum_{k=0}^{\infty}(-1)^{k}\left(\frac{x^{k}}{2^{k} k !}\right)^{2}
$$

is the zero-order Bessel function of the first kind, $f_{D}$ is the maximum Doppler frequency that is experienced by the moving vehicle, and $\sigma_{g}^{2}$ is the variance of $\tilde{G}(t)$. At each signaling interval of length $T$, the demodulator forms two decision variables $\{0,1\}$ and decides which signal was more likely to have been transmitted. A general block diagram for visualizing the behavior of such systems is given in Fig. 1 .

The combination of digital modulator, fading channel, and digital demodulator yields the equivalent DCCA model. The study and the analysis of the statistical behavior of the DCCA model are important since the design and the construction of effective error control schemes for this simplified (binary input, binary output) model help us better exploit the system memory and achieve reliable communication over the underlying correlated fading channel.

The DCCA is represented as an additive noise channel with binary error process $\left\{Z_{n}\right\}_{n=1}^{\infty}$, where $Z_{n}$ is as shown at the bottom of the page. The probability of an error sequence of

$$
L_{\omega}^{(M)}=\frac{\prod_{j=0}^{M-1-\omega}\left[j \frac{\varepsilon}{M-1+\alpha}+(1-\varepsilon)(1-p)\right] \prod_{j=0}^{\omega-1}\left[j \frac{\varepsilon}{M-1+\alpha}+(1-\varepsilon) p\right]}{\prod_{j=0}^{M-1}\left[1-(\alpha+j) \frac{\varepsilon}{M-1+\alpha}\right]}
$$

$$
Z_{n}= \begin{cases}0, & \text { if the } n \text {th transmitted symbol is correctly received } \\ 1, & \text { if the } n \text {th transmitted symbol is incorrectly received }\end{cases}
$$


length $n, z^{n}=\left(z_{1}, z_{2}, \ldots, z_{n}\right)$, can be directly obtained from [46, eq. (3), with, Omega, 1], i.e.,

$$
\begin{aligned}
P_{\mathrm{DCCA}}\left(z^{n}\right) \triangleq & \operatorname{Pr}\left\{Z^{n}=z^{n}\right\} \\
= & \sum_{l_{1}=z_{1}}^{1} \cdots \sum_{l_{n}=z_{n}}^{1}\left(\prod_{k=1}^{n} \frac{(-1)^{l_{k}+z_{k}}}{l_{k}+1}\right) \\
& \times \frac{\exp \left\{-\frac{E_{s}}{N_{0}} K_{R} \mathbf{1}^{T} \boldsymbol{F}\left(\left(K_{R}+1\right) \boldsymbol{I}+\frac{E_{s}}{N_{0}} \overline{\boldsymbol{C}} \boldsymbol{F}\right)^{-1} \mathbf{1}\right\}}{\operatorname{det}\left(\boldsymbol{I}+\frac{E_{s}}{N_{0}}\left(1+K_{R}\right)^{-1} \overline{\boldsymbol{C}} \boldsymbol{F}\right)}
\end{aligned}
$$

where $\boldsymbol{F}$ is a diagonal matrix, which is defined as

$$
\boldsymbol{F}=\operatorname{diag}\left(\frac{l_{1}}{l_{1}+1}, \ldots, \frac{l_{n}}{l_{n}+1}\right) .
$$

$\boldsymbol{I}$ is the identity matrix, $K_{R}=\eta / 2 \sigma_{g}^{2}$ is the Rician factor, and $\overline{\boldsymbol{C}}$ is the normalized covariance matrix with entries

$\bar{C}_{i j}=\left(1 / \sigma_{g}^{2}\right) \operatorname{Cov}(|i-j|)=J_{0}\left(2 \pi f_{D} T|i-j|\right), \quad 1 \leq i, j \leq n$.

The QBC is next used to model the equivalent binary error sequence of the DCCA, which represents the successes and failures that result from the transmission of symbols over the above fading channel.

\section{B. Estimation of Channel Parameters}

1) $Q B C$ Parameter Estimation: For a given DCCA, we construct a QBC whose noise or error process is statistically "close" in the Kullback-Leibler sense to the noise process that is generated by the DCCA. The Kullback-Leibler distance or divergence is an approximation quality measure that is widely used to determine the statistical closeness between two sources (e.g., see [6], [11], and [49]). Specifically, given a DCCA with fixed average SNR $E_{s} / N_{0}$, normalized Doppler frequency $f_{D} T$, and Rician factor $K_{R}$ resulting in BER DCCA $_{\text {and corre- }}$ lation coefficient $\operatorname{Cor}_{\mathrm{DCCA}}$, we estimate the QBC parameters $M, p, \varepsilon$, and $\alpha$ that minimize the KLDR, i.e.,

$$
\lim _{n \rightarrow \infty} \frac{1}{n} D_{n}\left(P_{\mathrm{DCCA}} \| P_{\mathrm{QBC}}^{(M)}\right)
$$

subject to the constraints

$$
\mathrm{BER}_{\mathrm{QBC}}=\mathrm{BER}_{\mathrm{DCCA}} \text { and } \operatorname{Cor}_{\mathrm{QBC}}=\mathrm{Cor}_{\mathrm{DCCA}}
$$

where $D_{n}\left(P_{\mathrm{DCCA}} \| P_{\mathrm{QBC}}^{(M)}\right)$ is the Kullback-Leibler divergence between the $n$-fold DCCA and QBC noise distributions, $\mathrm{P}_{\mathrm{DCCA}}$ and $\mathrm{P}_{\mathrm{QBC}}^{(M)}$, respectively, i.e.,

$$
D_{n}\left(P_{\mathrm{DCCA}} \| P_{\mathrm{QBC}}^{(M)}\right) q=\sum_{z^{n} \epsilon\{0,1\}^{n}} P_{\mathrm{DCCA}}\left(z^{n}\right) \log _{2} \frac{P_{\mathrm{DCCA}}\left(z^{n}\right)}{P_{\mathrm{QBC}}^{(M)}\left(z^{n}\right)}
$$

where $P_{\mathrm{QBC}}^{(M)}$ is given in closed form by (4) and (5), and $P_{\mathrm{DCCA}}$ is given by (11). Note that we focus on minimizing the KLDR, which is an asymptotic quantity [as opposed to minimizing the normalized divergence $(1 / n) D_{n}\left(P_{\mathrm{DCCA}} \| P_{\mathrm{QBC}}^{(M)}\right)$ for finite $n$ ], since it is vital to have identical statistical behavior on both channels for large block lengths (as large block lengths are required to achieve reliable communication by the channel coding theorem [49]).

It can be shown (e.g., see [53]) that the KLDR between the DCCA noise process (which is stationary) and the QBC noise process (which is Markovian) does exist and can be expressed as

$$
\begin{aligned}
\lim _{n \rightarrow \infty} \frac{1}{n} D_{n}\left(P_{\mathrm{DCCA}} \| P_{\mathrm{QBC}}^{(M)}\right)=-\mathcal{H}_{\mathrm{DCCA}}(Z)-E_{P_{\mathrm{DCCA}}} \\
\times\left[\log _{2} P_{\mathrm{QBC}}^{(M)}\left(Z_{M+1} \mid Z^{M}\right)\right]
\end{aligned}
$$

where $\mathcal{H}(Z) \triangleq \lim _{n \rightarrow \infty}(1 / n) H\left(Z^{n}\right)$ denotes the entropy rate [49]

$$
\begin{aligned}
E_{P_{\mathrm{DCCA}}}\left[\log _{2} \mathrm{P}_{\mathrm{QBC}}^{(M)}\left(Z_{M+1} \mid Z^{M}\right)\right] & \\
\triangleq & \sum_{z^{M+1}} P_{\mathrm{DCCA}}\left(z^{M+1}\right)\left[\log _{2} P_{\mathrm{QBC}}^{(M)}\left(z_{M+1} \mid z^{M}\right)\right]
\end{aligned}
$$

and $P_{\mathrm{QBC}}^{(M)}\left(z_{M+1} \mid z^{M}\right)$ is the QBC conditional error probability of symbol $M+1$ given the previous $M$ symbols. Then, the above minimization reduces to maximizing the second term in (12) (which is independent of $n$ ) over the QBC parameters. Note that in our approximation, we match BER and Cor of both channels to guarantee identical noise marginal distributions and identical probabilities of two consecutive errors (ones). Hence, given these constraints, the above optimization problem reduces to an optimization over only two QBC parameters $-M$ and $\varepsilon$. This is numerically achieved by sequentially incrementing $M \geq 1$ and varying $0.0001 \leq \varepsilon \leq 0.9999$ for each given $M$.

2) GEC Parameter Estimation: We next briefly describe the method of modeling the DCCA via the GEC introduced by Pimentel et al. [46]. For a given DCCA, the parameterization of the GEC is based on the following lemma.

Lemma 1: The probability of any observed sequence $z^{n}$ generated by the GEC satisfies the following recurrence equation [46]:

$$
P_{\mathrm{GEC}}\left(z^{n} \varsigma \kappa\right)=c(\varsigma, \kappa) P_{\mathrm{GEC}}\left(z^{n} \varsigma\right)+d(\varsigma, \kappa) P_{\mathrm{GEC}}\left(z^{n}\right)
$$

where $P_{\mathrm{GEC}}\left(z^{n} \varsigma \kappa\right) \triangleq \operatorname{Pr}\left\{Z_{1}=z_{1}, \ldots, Z_{n}=z_{n}, Z_{n+1}=\varsigma\right.$, $\left.Z_{n+2}=\kappa\right\}, \varsigma$ and $\kappa$ are binary symbols, and

$$
\begin{aligned}
& c(0,0)=\left(1-p_{G}\right)(1-b)+\left(1-p_{B}\right)(1-g) \\
& c(1,1)=p_{G}(1-b)+p_{B}(1-g) \\
& d(0,0)=-(1-g-b)\left(1-p_{G}\right)\left(1-p_{B}\right) \\
& \left.d(1,1)=-(1-g-b) p_{G}\right) p_{B} \\
& c(1,0)=1-c(1,1), \quad c(0,1)=1-c(0,0) \\
& d(0,1)=-d(0,0), \quad \text { and } \quad d(1,0)=-d(1,1) .
\end{aligned}
$$

Lemma 1 shows that the parameters $c(\varsigma, \kappa)$ and $d(\varsigma, \kappa)$ can be calculated via a linear system of equations. For example, 
setting $z^{n}=\phi$, where $\phi$ is an empty sequence, $P_{\mathrm{GEC}}(\phi)=1$, and $z^{n}=\varsigma$ in (13), $c(\varsigma, \kappa)$ and $d(\varsigma, \kappa)$ can be determined by the probabilities of error sequences of length at most 3 , i.e.,

$$
c(\varsigma, \kappa)=\frac{P_{\mathrm{GEC}}(\varsigma \varsigma \kappa)-P_{\mathrm{GEC}}(\varsigma \kappa) P_{\mathrm{GEC}}(\varsigma)}{P_{\mathrm{GEC}}(\varsigma \varsigma)-P_{\mathrm{GEC}}^{2}(\varsigma)}
$$

and

$$
d(\varsigma, \kappa)=\frac{P_{\mathrm{GEC}}(\varsigma \kappa) P_{\mathrm{GEC}}(\varsigma \varsigma)-P_{\mathrm{GEC}}(\varsigma \varsigma \kappa) P_{\mathrm{GEC}}(\varsigma)}{P_{\mathrm{GEC}}(\varsigma \varsigma)-P_{\mathrm{GEC}}^{2}(\varsigma)} .
$$

The GEC parameters follow by solving the nonlinear equations in (14) and (15) as follows.

Proposition 1: If $P_{\mathrm{GEC}}(01) \neq P_{\mathrm{GEC}}(0) P_{\mathrm{GEC}}(1)$, the parameters of the GEC are uniquely determined by the four probabilities $P_{\mathrm{GEC}}(0), P_{\mathrm{GEC}}(00), P_{\mathrm{GEC}}(000)$, and $P_{\mathrm{GEC}}(111)$. The four parameters $b, g, p_{G}$, and $p_{B}$ are given by the following. $p_{G}$ and $p_{B}$ are the roots of the following quadratic equation [46]:

$$
\begin{aligned}
& {[-1+c(1,1)+c(0,0)] x^{2}} \\
& \quad+[1-c(1,1)-c(0,0)+d(1,1)-d(0,0)] x-d(1,1)=0
\end{aligned}
$$

and

$$
\begin{aligned}
& b=\frac{c(0,0) p_{B}-c(1,1)\left(1-p_{B}\right)+\left(p_{G}-p_{B}\right)}{p_{G}-p_{B}} \\
& g=\frac{c(0,0) p_{G}-c(1,1)\left(1-p_{G}\right)+\left(p_{B}-p_{G}\right)}{p_{B}-p_{G}} .
\end{aligned}
$$

Hence, if $P_{\mathrm{DCCA}}(0), P_{\mathrm{DCCA}}(00), P_{\mathrm{DCCA}}(000)$, and $P_{\mathrm{DCCA}}(111)$ are known, where $P_{\mathrm{DCCA}}\left(z^{n}\right)$ is the probability of error sequences generated by the DCCA [see (11)], the parameters of the GEC can be obtained by (16), (17), and Proposition 1 by setting $P_{\mathrm{GEC}}\left(z^{n}\right)=P_{\mathrm{DCCA}}\left(z^{n}\right), n=1,2$, and 3.

\section{Modeling Results And Discussions}

We evaluate how well the QBC model fits or approximates the DCCA according to two criteria: ACF and channel capacity. The QBC ACF and capacity expressions are provided in Section II-B. The ACF of the DCCA can be directly obtained from (11), i.e.,

$$
\begin{aligned}
R_{\mathrm{DCCA}}[m]= & \frac{\left(1+K_{R}\right)^{2}}{\left(2+2 K_{R}+\frac{E_{s}}{N_{0}}\right)^{2}-\left(\frac{E_{s}}{N_{0}} \rho(m)\right)^{2}} \\
& \times \exp \left\{-\frac{2 K_{R} \frac{E_{s}}{N_{0}}}{2+2 K_{R}+\frac{E_{S}}{N_{0}}(\rho(m)+1)}\right\}
\end{aligned}
$$

where $\rho(m)=J_{0}\left(2 \pi m f_{D} T\right)$.

As in (9), the capacity of the DCCA is given by

$$
C_{\mathrm{DCCA}}=\lim _{n \rightarrow \infty} \sup _{X^{n}} \frac{1}{n} I\left(X^{n} ; Y^{n}\right)=1-\mathcal{H}_{\mathrm{DCCA}}(Z) .
$$

The entropy rate $\mathcal{H}_{\mathrm{DCCA}}(Z)$ of the (stationary ergodic) DCCA error process is not known in closed form. However, we can approximate it by calculating the normalized block noise entropy

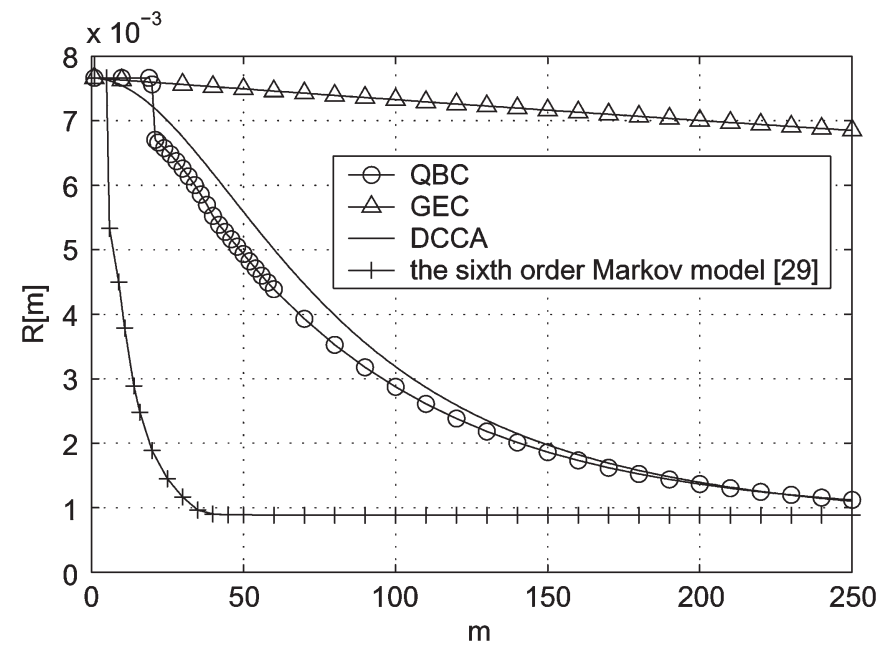

Fig. 2. DCCA fitting via the QBC: ACF versus $m$ for $f_{D} T=0.001$, SNR $=$ $15 \mathrm{~dB}$, and $K_{R}=-\infty \mathrm{dB}$ (Rayleigh).

$(1 / n) H\left(Z^{n}\right)$ for large values of $n$ and, thus, obtain a lower bound on $C_{\mathrm{DCCA}}$, which is given by

$$
C_{\mathrm{DCCA}} \geq C_{\mathrm{DCCA}, \mathrm{n}} \triangleq 1-\frac{1}{n} H_{\mathrm{DCCA}}\left(Z^{n}\right) .
$$

In our calculations, we used values of $n$ as large as 21 .

For the sake of comparison, we also present modeling results via the GEC using the method of Pimentel et al. [46] (which we briefly described in Section III-B2). Note that in [46], the authors also employ arbitrary $K$ th-order Markov noise models to approximate the fading channels. However, unlike our QBC model, which has only four parameters (as the GEC) and allows large values for its memory order $M$ (since its noise is a specially structured $M$ th-order Markov process that is generated by our queue scheme), the $K$ th-order Markov models of [46] are unstructured and, hence, suffer from the limitation of having a number of parameters that exponentially grow $^{3}$ with $K$. Therefore, with the exception of a brief comparison with the Markov model of [46] (see Fig. 2), we herein mainly compare our QBC-based modeling method with the GEC-based modeling method of [46] since both channels have identical number of parameters and, hence, identical degrees of freedom and complexity.

The capacity of the GEC is obtained via the algorithm in [30]. The ACF of the GEC can be also directly obtained from (1), i.e.,

$$
R_{\mathrm{GEC}}[m]=\boldsymbol{\pi}^{T} \boldsymbol{P}(1)\left(\prod_{k=1}^{m-1} \boldsymbol{P}\right) \boldsymbol{P}(1) \mathbf{1}
$$

where $\boldsymbol{\pi}, \boldsymbol{P}(1)$, and $\boldsymbol{P}$ are defined in Section II-A.

A wide range of DCCA channel parameters is investigated with SNR $=15$ and $25 \mathrm{~dB}, f_{D} T=0.001,0.005,0.01$, and 0.1 for Rayleigh fading $\left(K_{R}=-\infty \mathrm{dB}\right)$, and $\mathrm{SNR}=15 \mathrm{~dB}$ and

\footnotetext{
${ }^{3}$ As a result, only models with memory order up to six are studied in [46]. Such models are shown to well approximate channels with fast and medium fading rates $\left(f_{D} T>0.2\right)$; however, they are inadequate for slow fading rates. As we later show in this section, the QBC model can accommodate large values of the memory order; thus, it can provide a good approximation of channels with slow fading $\left(f_{D} T<0.02\right)$ in addition to medium and fast fading.
} 
TABLE I

QBC AND GEC MODELING PARAMETERS FOR

$K_{R}=-\infty \mathrm{dB}(\mathrm{RAYLEIGH}) \mathrm{AND}$ SNR $=15 \mathrm{~dB}$

\begin{tabular}{|c|l|l|l|l|}
\hline \hline \multirow{2}{*}{ Model } & \multicolumn{4}{|c|}{$f_{D} T$} \\
\cline { 2 - 5 } & \multicolumn{1}{|c|}{0.001} & \multicolumn{1}{|c|}{0.005} & \multicolumn{1}{c|}{0.01} & \multicolumn{1}{c|}{0.1} \\
\hline \multirow{3}{*}{ QBC } & $M=20$ & $M=11$ & $M=7$ & $M=2$ \\
& $\varepsilon=0.8593$ & $\varepsilon=0.7602$ & $\varepsilon=0.6556$ & $\varepsilon=0.0893$ \\
& $p=0.0297$ & $p=0.0297$ & $p=0.0297$ & $p=0.0297$ \\
& $\alpha=0.8959$ & $\alpha=0.3828$ & $\alpha=0.3387$ & $\alpha=0.131$ \\
\hline \multirow{4}{\text{GEC}}{} & $b=0.0000339$ & $b=0.000841$ & $b=0.00329$ & $b=0.0324$ \\
& $g=0.000479$ & $g=0.0118$ & $g=0.045$ & $g=0.7466$ \\
& $p_{B}=0.3393$ & $p_{B}=0.3393$ & $p_{B}=0.3395$ & $p_{B}=0.5199$ \\
& $p_{G}=0.00783$ & $p_{G}=0.00766$ & $p_{G}=0.00713$ & $p_{G}=0.00849$ \\
\hline \hline
\end{tabular}

TABLE II

QBC AND GEC MODELING PARAMETERS FOR

$K_{R}=-\infty \mathrm{dB}$ (RAYLEIGH) AND SNR $=25 \mathrm{~dB}$

\begin{tabular}{|c|l|l|l|l|}
\hline \hline \multirow{2}{*}{ Model } & \multicolumn{4}{|c|}{$f_{D} T$} \\
\cline { 2 - 5 } & \multicolumn{1}{|c|}{0.001} & \multicolumn{1}{|c|}{0.005} & \multicolumn{1}{|c|}{0.01} & \multicolumn{1}{c|}{0.1} \\
\hline \multirow{3}{*}{ QBC } & $M=18$ & $M=6$ & $M=4$ & $M=2$ \\
& $\varepsilon=0.8506$ & $\varepsilon=0.6226$ & $\varepsilon=0.4666$ & $\varepsilon=0.0145$ \\
& $p=0.00314$ & $p=0.00314$ & $p=0.00314$ & $p=0.00314$ \\
& $\alpha=0.2607$ & $\alpha=0.2525$ & $\alpha=0.2019$ & $\alpha=0.1054$ \\
\hline \multirow{4}{*}{ GEC } & $b=0.0000333$ & $b=0.000773$ & $b=0.0025$ & $b=0.00103$ \\
& $g=0.00466$ & $g=0.1014$ & $g=0.2887$ & $g=0.8338$ \\
& $p_{B}=0.3339$ & $p_{B}=0.334$ & $p_{B}=0.3343$ & $p_{B}=0.4523$ \\
& $p_{G}=0.000783$ & $p_{G}=0.000622$ & $p_{G}=0.000279$ & $p_{G}=0.00259$ \\
\hline \hline
\end{tabular}

TABLE III

QBC AND GEC MODELING PARAMETERS FOR

$K_{R}=5 \mathrm{~dB}$ (RICIAN) AND SNR $=15 \mathrm{~dB}$

\begin{tabular}{|l|l||l|l|l|}
\hline \hline \multirow{3}{*}{ Model } & \multicolumn{4}{|c|}{$f_{D} T$} \\
\cline { 2 - 5 } & \multicolumn{1}{|c|}{0.001} & \multicolumn{1}{|c|}{0.005} & \multicolumn{1}{c|}{0.01} & \multicolumn{1}{c|}{0.05} \\
\hline \multirow{3}{*}{ QBC } & $M=18$ & $M=17$ & $M=11$ & $M=3$ \\
& $\varepsilon=0.8195$ & $\varepsilon=0.8054$ & $\varepsilon=0.7219$ & $\varepsilon=0.3426$ \\
& $p=0.00853$ & $p=0.00853$ & $p=0.00853$ & $p=0.00853$ \\
& $\alpha=0.9619$ & $\alpha=0.3971$ & $\alpha=0.3299$ & $\alpha=0.3726$ \\
\hline \multirow{4}{*}{ GEC } & $b=0.00000259$ & $b=0.0000646$ & $b=0.000257$ & $b=0.00542$ \\
& $g=0.000139$ & $g=0.00347$ & $g=0.0137$ & $g=0.2533$ \\
& $p_{B}=0.3112$ & $p_{B}=0.3113$ & $p_{B}=0.3115$ & $p_{B}=0.3185$ \\
& $p_{G}=0.00289$ & $p_{G}=0.00288$ & $p_{G}=0.00284$ & $p_{G}=0.0019$ \\
\hline \hline
\end{tabular}

$f_{D} T=0.001,0.005,0.01$, and 0.05 for Rician fading $\left(K_{R}=\right.$ $5 \mathrm{~dB}$ ). The SNR, $f_{D} T$, and $K_{R}$ values (except for $f_{D} T=$ $0.005)$ were chosen to match the conditions of the correlated Rician and Rayleigh fading channels studied in [46, Figs. 6, 7, 9, and 11]. The QBC and GEC parameters, which were obtained as explained in Sections III-B1 and 2, respectively, are provided in Tables I-III.

A subset of the modeling results in terms of the ACF for the DCCA, its QBC approximation, and its GEC approximation is shown in Figs. 2-5 (the complete results are available in [45]). We observe a strong ACF agreement between the QBC and the DCCA in these figures. ${ }^{4}$ This behavior is indeed observed for all computations, particularly for $f_{D} T=0.1$, where the

${ }^{4}$ Note that the $\mathrm{ACF}$ of the $\mathrm{QBC} R_{\mathrm{QBC}}[m]$ is equal to a constant for $m \leq M-1$, as indicated by (8).

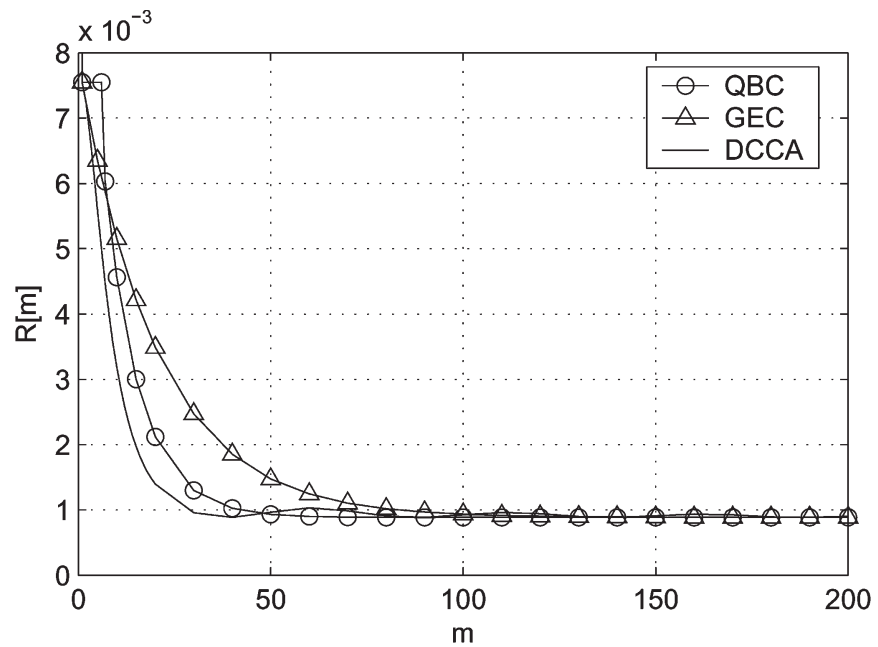

Fig. 3. DCCA fitting via the QBC: ACF versus $m$ for $f_{D} T=0.01$, SNR $=$ $15 \mathrm{~dB}$, and $K_{R}=-\infty \mathrm{dB}$ (Rayleigh).

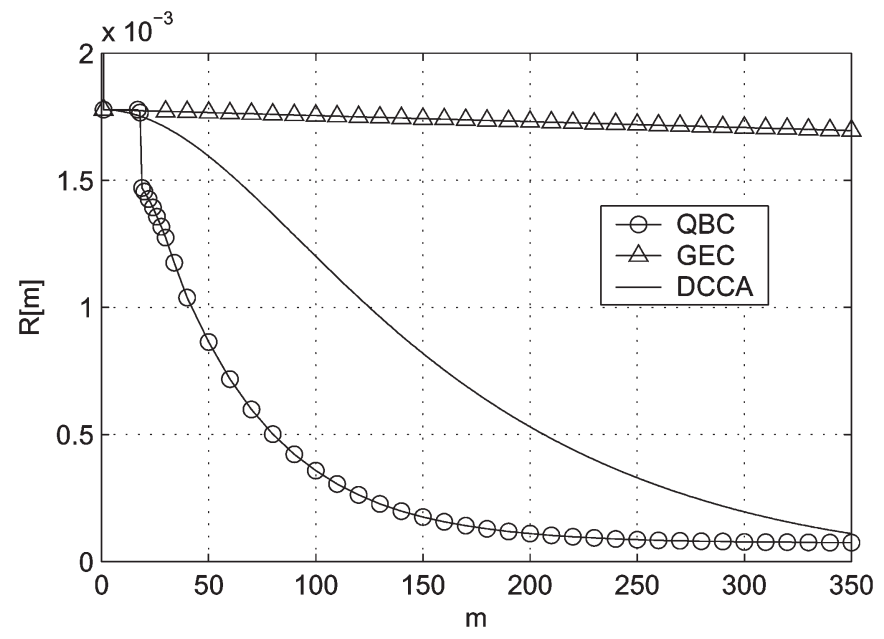

Fig. 4. DCCA fitting via the QBC: ACF versus $m$ for $f_{D} T=0.001$, SNR $=$ $15 \mathrm{~dB}$, and $K_{R}=5 \mathrm{~dB}$ (Rician).

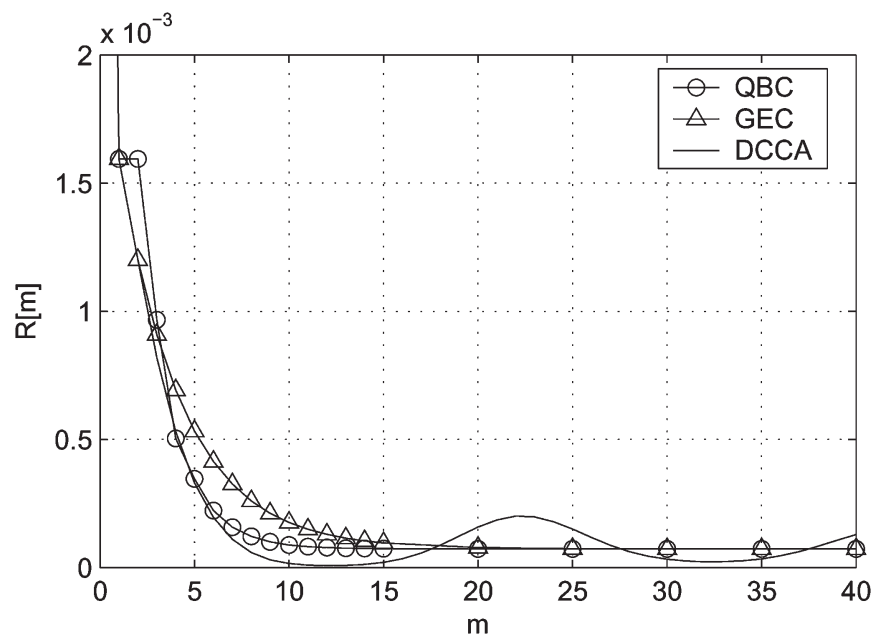

Fig. 5. DCCA fitting via the QBC: ACF versus $m$ for $f_{D} T=0.05$, SNR $=$ $15 \mathrm{~dB}$, and $K_{R}=5 \mathrm{~dB}$ (Rician). 


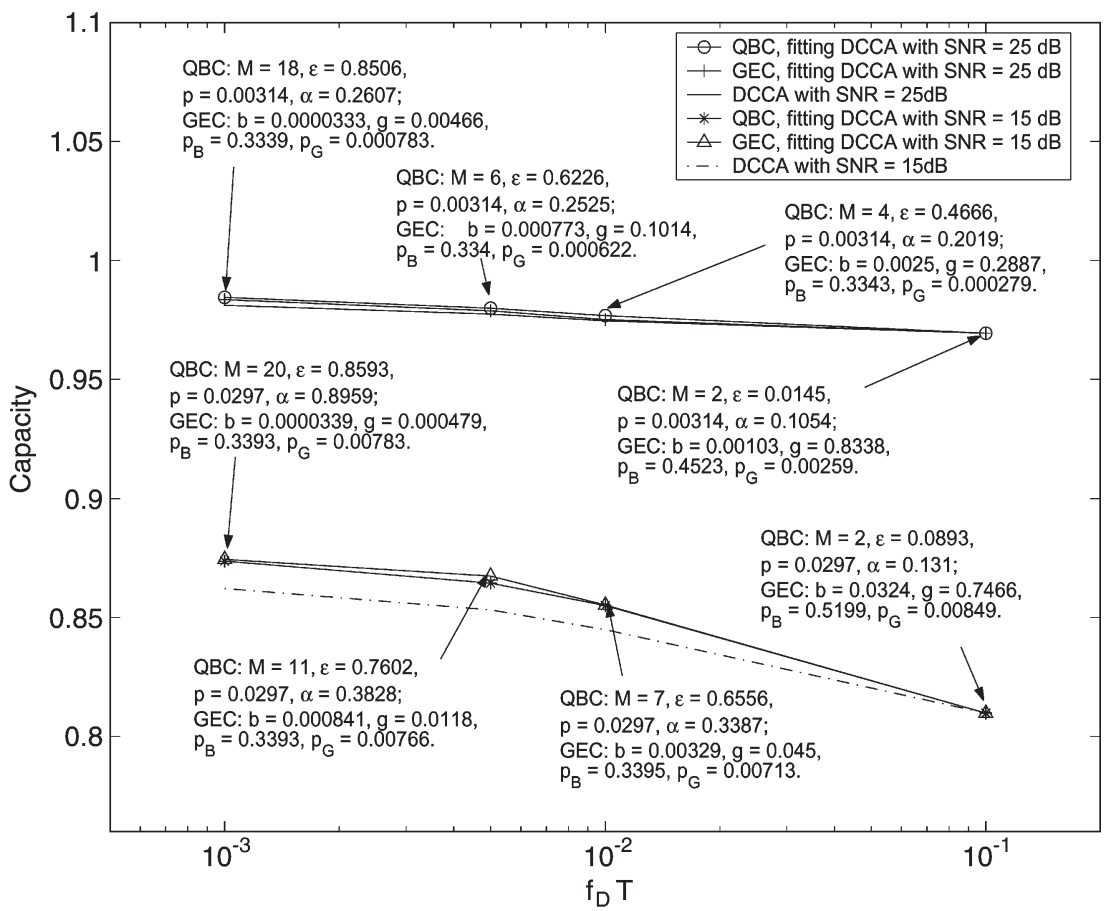

Fig. 6. DCCA fitting via the QBC. Capacity (in bits per channel use) versus normalized Doppler frequency $f_{D} T$ for Rayleigh fading.

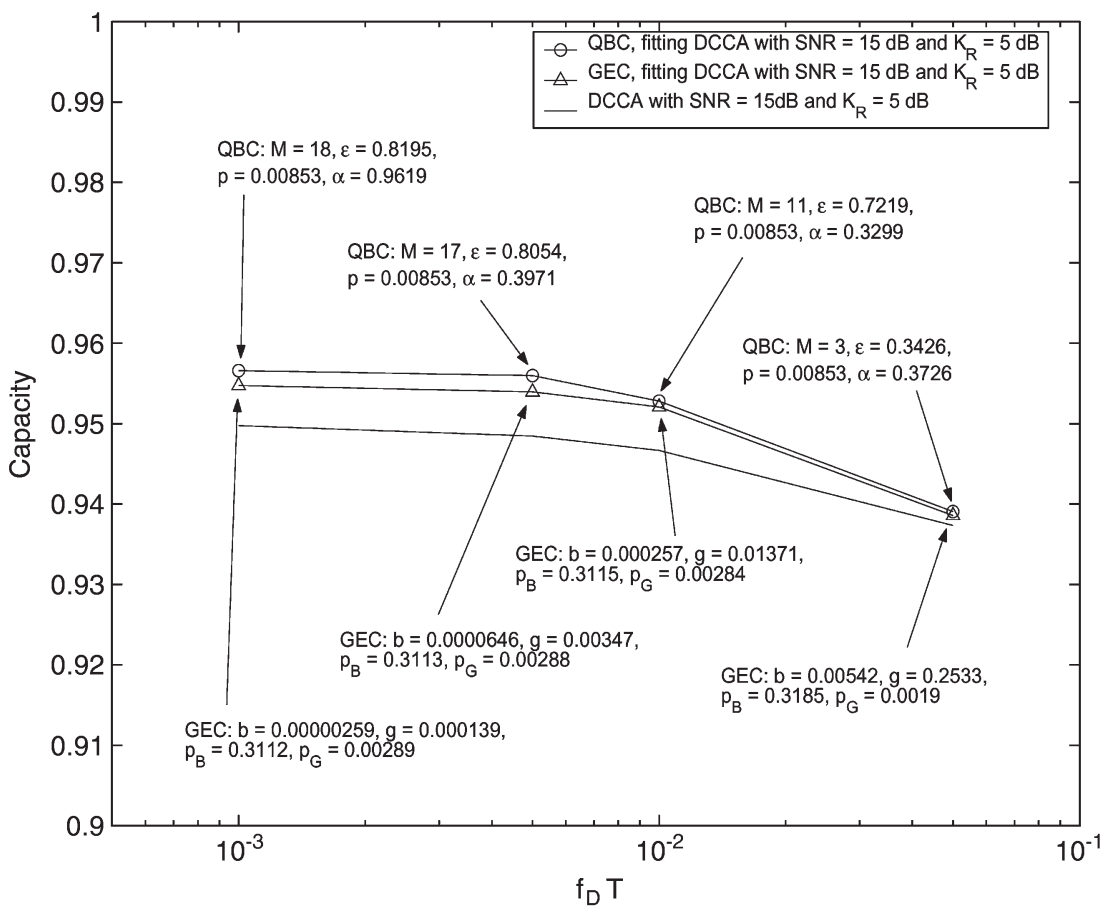

Fig. 7. DCCA fitting via the QBC. Capacity (in bits per channel use) versus normalized Doppler frequency $f_{D} T$ for Rician fading.

ACF curves of the DCCA and its QBC approximation are identical [45]. For slow and medium fading (e.g., see Fig. 2 and [45]), the ACF curve for the GEC takes a longer span of $m$ before eventually converging, which indicates that the GEC (as fitted in [46]) might not be adequate for modeling very slow Rayleigh fading $\left(f_{D} T=0.001\right)$ and very slow to medium Rician fading $\left(f_{D} T=0.001,0.005\right.$, and 0.01$)$. We observe that the QBC has a better performance than the Markov models in [46] (see Fig. 2) but with significantly smaller complexity since it is fully described by four parameters and allows closed- form expressions for various fading characteristics. Compared with [46, Fig. 7(a)], the QBC has similar performance as the Markov models of [46] with order 4 or 5 but with smaller complexity.

Note that since the QBC noise is a homogeneous Markov process, the KLDR between the DCCA and QBC error processes exists and admits a simple expression given by (12). Hence, it is practical to minimize this KLDR by maximizing the expected value in (12) over the QBC parameters, which is independent of $n$ (see Section III-B1). However, this approach 
is not easily applicable to the GEC since the KLDR between the DCCA and GEC noise processes does not admit a simple expression in general (as the GEC noise is hidden Markovian). The method of parameterization of the GEC of Section III-B2 is simple; however, it only takes into account error sequences no longer than three, which implies that this method is not appropriate for approximating slow fading.

Our results show that the largest Markovian memory $M$ for the QBC model that best fits the DCCA is 20 , whereas the largest Markovian memory $K$ for the (unstructured) Markov noise channel models considered in [46] is 6 (higher order unstructured models could not be obtained in [46] due to their prohibitive exponential complexity). This explains why the QBC is more suitable for fitting slow fading with large memory than the Markov noise model considered in [46].

Modeling results in terms of capacity are shown in Figs. 6 and 7, where the lower bound for the capacity of the DCCA and the capacities of the QBC approximation and the GEC approximation are shown for different SNR and $f_{D} T$ values. We clearly observe from the figures that the capacity curves of the QBC and the lower bound curves for the capacity of the DCCA match quite well, and the capacities for $f_{D} T=0.1$ (fast Rayleigh fading) are almost identical. The last observation can be explained by the fact that the DCCA has low memory at $f_{D} T=0.1$ (fast fading); hence, the lower bound for its capacity is tight [since $(1 / n) H\left(Z^{n}\right)=H\left(Z_{1}\right)$ if $Z^{n}$ is memoryless]. Overall, we observe a strong match in capacity between the DCCA and its QBC approximation. In terms of capacity, the GEC has nearly as good a performance as the QBC in fitting the DCCA.

\section{SUMmary}

In this paper, we approximate hard-decision demodulated correlated Rician fading channels (represented by the DCCA model) via the QBC model. Numerical results show a strong agreement between the ACF and capacity curves of the QBC and the DCCA. This leads us to conclude that the QBC provides a very good approximation of the DCCA under a variety of channel conditions. The QBC provides a much better performance in terms of ACF for fitting the DCCA than the GEC and the Markov models of [46] for the range of slow and very slow fading. An important feature of this QBC model is that it is valuable for characterizing a wide class of communication channels with memory while remaining mathematically simple and flexible.

One possible direction for future work is the modeling and analysis of wired/wireless Internet traffic and channel coding as an extension and application of this paper. Sanneck and Carle [54] used an $M$ th-order Markov chain process to describe the dependencies between packet losses. However, their models have a complexity (number of parameters) that exponentially grows with $M$, rendering it impractical for the modeling of packet loss processes with large memory. The QBC model, on the other hand, does not suffer from this limitation, as it is fully described by only four parameters and allows single-letter analysis. The QBC, hence, can be employed to characterize the packet-loss patterns introduced by the Internet, particularly to capture loss burstiness and distances between loss bursts. Another topic of future interest is the design, construction, and analysis of channel codes for the QBC. One important objective in this problem is the judicious design of the powerful channel codes to fully exploit the channel memory. Some results in this direction involving low-density parity check codes are reported in [55].

\section{REFERENCES}

[1] L. Zhong, F. Alajaji, and G. Takahara, "A queue-based model for wireless Rayleigh fading channels with memory," in Proc. IEEE 62nd Semiannual Veh. Technol. Conf._Fall, Dallas, TX, Sep. 2005, pp. 1362-1366.

[2] L. N. Kanal and A. R. K. Sastry, "Models for channels with memory, their applications to error control," Proc. IEEE, vol. 66, no. 7, pp. 724-744, Jul. 1978.

[3] L. Wilhelmsson and L. B. Milstein, "On the effect of imperfect interleaving for the Gilbert-Elliott channel," IEEE Trans. Commun., vol. 47, no. 5, pp. 681-688, May 1999.

[4] H. Bischl and E. Lutz, "Packet error rate in the non-interleaved Rayleigh channel," IEEE Trans. Commun., vol. 43, no. 2-4, pp. 1375-1382, Feb.-Apr. 1995.

[5] C. Pimentel and I. F. Blake, "Non-interleaved Reed-Solomon coding performance on finite state channels," in Proc. IEEE Int. Conf. Commun., 1997, vol. 3, pp. 1493-1497.

[6] C. Pimentel and I. F. Blake, "Modeling burst channels using partitioned Fritchman's Markov models," IEEE Trans. Veh. Technol., vol. 47, no. 3, pp. 885-899, Aug. 1998.

[7] J. G. Proakis, Digital Communications, 4th ed. New York: McGrawHill, 2000.

[8] C. C. Tan and N. C. Beaulieu, "Infinite series representations of the bivariate Rayleigh and Nakagami- $m$ distribution," IEEE Trans. Commun., vol. 45, no. 10, pp. 1159-1161, Oct. 1997.

[9] R. G. Gallager, Information Theory and Reliable Communication. New York: Wiley, 1968.

[10] A. Lapidoth and P. Narayan, "Reliable communication under channel uncertainty," IEEE Trans. Inf. Theory, vol. 44, no. 6, pp. 2148-2177, Oct. 1998.

[11] W. Turin, Performance Analysis of Digital Transmission Systems. New York: Comput. Sci., 1990.

[12] E. N. Gilbert, "Capacity of a burst-noise channel," Bell Syst. Tech. J., vol. 39, pp. 1253-1266, Sep. 1960.

[13] E. O. Elliott, "Estimates of error rates for codes on burst-noise channel," Bell Syst. Tech. J., vol. 42, pp. 1977-1997, Sep. 1963.

[14] B. D. Fritchman, "A binary channel characterization using partitioned Markov chains," IEEE Trans. Inf. Theory, vol. IT-13, no. 2, pp. 221-227, Apr. 1967.

[15] S. Tsai, "Markov characterization of the HF channel," IEEE Trans. Commun., vol. COM-17, no. 1, pp. 24-32, Feb. 1969.

[16] L. Ahlin, "Coding methods for the mobile radio channel," in Proc. Nordic Semin. Digital Land Mobile Commun., Espoo, Finland, Feb. 1985, pp. 185-194.

[17] J.-Y. Chouinard, M. Lecours, and G. Y. Delisle, "Estimation of Gilbert's and Fritchman's models parameters using the gradient method for digital mobile radio channels," IEEE Trans. Veh. Technol., vol. 37, no. 3, pp. 158166, Aug. 1988.

[18] F. Swarts and H. C. Ferreira, "Markov characterization of digital fading mobile VHF channels," IEEE Trans. Veh. Technol., vol. 43, no. 4, pp. 977985, Nov. 1994

[19] V. Y. Y. Chu and P. Sweeney, "Characterizing error sequences of low Earth orbit satellite channel and optimization with hybrid-ARQ schemes," in Proc. GLOBECOM, Sydney, Australia, Nov. 1998, vol. 5, pp. 2930-2935.

[20] A. I. Drukarev and K. P. Yiu, "Performance of error-correcting codes on channels with memory," IEEE Trans. Commun., vol. COM-34, no. 6, pp. 513-521, Jun. 1986.

[21] K. Sakakibara, "Performance analysis of the error-forecasting decoding for interleaved block codes on Gilbert-Elliott channels," IEEE Trans. Commun., vol. 48, no. 3, pp. 386-395, Mar. 2000.

[22] G. Sharma, A. A. Hassan, and A. Dholakia, "Performance evaluation of burst-error-correcting codes on a Gilbert-Elliott channel," IEEE Trans. Commun., vol. 46, no. 7, pp. 846-849, Jul. 1998.

[23] H. S. Wang and N. Moayeri, "Finite-state Markov channel-A useful model for radio communication channels," IEEE Trans. Veh. Technol., vol. 44, no. 1, pp. 163-171, Feb. 1995. 
[24] C. C. Tan and N. C. Beaulieu, "On first-order Markov modeling for the Rayleigh fading channel," IEEE Trans. Commun., vol. 48, no. 12, pp. 2032-2040, Dec. 2000.

[25] Q. Zhang and S. Kassam, "Finite state Markov model for Rayleigh fading channels," IEEE Trans. Commun., vol. 47, no. 11, pp. 1688-1692, Nov. 1999.

[26] M. Zorzi, R. R. Rao, and L. B. Milstein, "ARQ error control on fading mobile radio channels," IEEE Trans. Veh. Technol., vol. 46, no. 2, pp. 445455, May 1997.

[27] M. Zorzi and R. R. Rao, "On the statistics of block errors in bursty channels," IEEE Trans. Commun., vol. 45, no. 6, pp. 660-667, Jun. 1997.

[28] M. J. Chu and W. E. Stark, "Effect of mobile velocity on communications in fading channels," IEEE Trans. Veh. Technol., vol. 49, no. 1, pp. 202210, Jan. 2000.

[29] W. Turin and R. van Nobelen, "Hidden Markov modeling of flat fading channels," IEEE J. Sel. Areas Commun., vol. 16, no. 9, pp. 1809-1817, Dec. 1998

[30] M. Mushkin and I. Bar-David, "Capacity, coding for the Gilbert-Elliott channel," IEEE Trans. Inf. Theory, vol. 35, no. 6, pp. 1277-1290, Nov. 1989.

[31] R. L. Dobrushin and M. S. Pinsker, "Memory increases transmission capacity," Probl. Peredachi Inf., vol. 5, no. 1, pp. 94-95, 1969.

[32] F. Alajaji and T. Fuja, "A communication channel modeled on contagion," IEEE Trans. Inf. Theory, vol. 40, no. 6, pp. 2035-2041, Nov. 1994.

[33] G. Polya, "Sur quelques points de la theorie des probabilites," Ann. Inst. Henri Poincare, vol. 1, pp. 117-161, 1931.

[34] F. Alajaji, N. Phamdo, N. Farvardin, and T. Fuja, "Detection of binary Markov sources over channels with additive Markov noise," IEEE Trans. Inf. Theory, vol. 42, no. 1, pp. 230-239, Jan. 1996.

[35] N. Phamdo, F. Alajaji, and N. Farvardin, "Quantization of memoryless, Gauss-Markov sources over binary Markov channels," IEEE Trans. Commun., vol. 45, no. 6, pp. 668-675, Jun. 1997.

[36] R. Iordache and I. Tabus, "Index assignment for transmitting vector quantized LSF parameters over binary Markov channels," in Proc. IEEE Int. Symp. Circuits Syst., Orlando, FL, May 1999, vol. 4, pp. 544-547.

[37] R. Iordache, I. Tabus, and J. Astola, "Robust index-assignment using Hadamard transform for vector quantization transmission over finitememory contagion channels," Circuits Syst. Signal Process., vol. 21, no. 5, pp. 485-509, Sep./Oct. 2002.

[38] K. P. Subbalakshmi and J. Vaisey, "On the joint source-channel decoding of variable-length encoded sources: The additive-Markov case," IEEE Trans. Commun., vol. 51, no. 9, pp. 1420-1425, Sep. 2003

[39] T. P.-C. Chen and T. Chen, "Error concealment aware rate shaping for wireless video transport," Signal Process. Image Commun., vol. 18, no. 10, pp. 889-905, Nov. 2003.

[40] Q. Chen and K. P. Subbalakshmi, "Joint source-channel decoding for MPEG-4 coded video over wireless channels," IEEE J. Sel. Areas Commun., vol. 21, no. 10, pp. 1780-1789, Dec. 2003.

[41] V. Nagarajan and O. Milenkovic, "Performance analysis of structured LDPC codes in the Polya-urn channel with finite memory," in Proc. IEEE Can. Conf. Elect. Comput. Eng., Niagara Falls, ON, Canada, May 2004, vol. 1 , pp. 543-546.

[42] F. Behnamfar, F. Alajaji, and T. Linder, "Image transmission over the Polya channel via channel-optimized quantization," IEEE Trans. Signal Process., vol. 53, no. 2, pp. 728-733, Feb. 2005.

[43] L. Zhong, F. Alajaji, and G. Takahara, "A queue-based model for binary communication channels," in Proc. Allerton Conf. Commun., Control, Comput., Monticello, IL, Oct. 2003, pp. 130-139. [Online]. Available: http://www.mast.queensu.ca/ web/publications.html

[44] L. Zhong, F. Alajaji, and G. Takahara, "A binary communication channel with memory based on a finite queue," IEEE Trans. Inf. Theory, vol. 53, no. 8, pp. 2815-2840, Aug. 2007.

[45] L. Zhong, "A binary burst-noise communication channel modeled by a finite queue: Information theoretic properties and applications to wireless communications," Ph.D. dissertation, Queen's Univ., Kingston, ON, Canada, Jun. 2005. [Online]. Available: http://www.mast. queensu.ca/ web/publications.html

[46] C. Pimentel, T. H. Falk, and L. Lisbôa, "Finite-state Markov modeling of correlated Rician fading channels," IEEE Trans. Veh. Technol., vol. 53, no. 5, pp. 1491-1501, Sep. 2004.

[47] L. Zhong, F. Alajaji, and G. Takahara, "An approximation of the Gilbert-Elliott channel via a queue-based channel model," in Proc. IEEE Int. Symp. Inf. Theory, Chicago, IL, Jun. 2004, p. 63.

[48] S. Verdú and T. S. Han, "A general formula for channel capacity," IEEE Trans. Inf. Theory, vol. 40, no. 4, pp. 1147-1157, Jul. 1994.

[49] T. M. Cover and J. A. Thomas, Elements of Information Theory. New York: Wiley, 1991.
[50] D. Kim, L. R. Welch, and R. E. Peile, "Correlation effect to Reed Solomon code performance and experiment of error/erasure decoder co-operating with interleaver and hidden Markov modeler on satellite channel," in Proc. IEEE MILCOM Conf., San Diego, CA, Oct. 1992, pp. 584-588.

[51] R. H. Clarke, "A statistical theory of mobile radio reception," Bell Syst. Tech. J., vol. 47, no. 6, pp. 957-1000, 1968.

[52] M. Gans, "A power spectral theory of propagation in the mobile radio environment," IEEE Trans. Veh. Technol., vol. VT-21, no. 1, pp. 27-38, Feb. 1972.

[53] R. M. Gray, Entropy and Information Theory. New York: SpringerVerlag, 1990

[54] H. Sanneck and G. Carle, "A framework model for packet loss metrics based on loss runlengths," in Proc. SPIE/ACM SIGMM Multimedia Comput. Netw. Conf., San Jose, CA, Jan. 2000, pp. 177-187.

[55] C. Nicola, F. Alajaji, and T. Linder, "Decoding LDPC codes over binary channels with additive Markov noise," in Proc. Can. Workshop Inf. Theory, Montreal, QC, Canada, Jun. 2005, pp. 187-190.

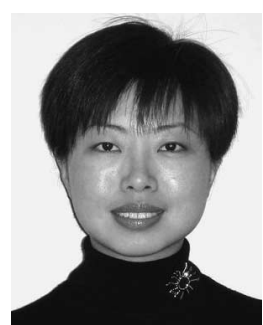

Libo Zhong received the B.Sc. degree from the University of Science and Technology of China, Hefei, China, in 1995 and the M.Sc. and Ph.D. degrees in applied mathematics from Queen's University, Kingston, ON, Canada, in 2001 and 2005, respectively.

She was a Research Scientist at Entrust, Ottawa, ON, during 2005 and 2006. In December 2006, she joined SPSS as a statistician.

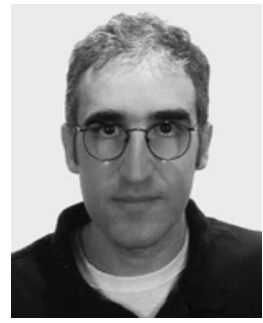

Fady Alajaji (S'90-M'94-SM'00) was born in Beirut, Lebanon, on May 1, 1966. He received the B.E. degree (with Distinction) from the American University of Beirut and the M.Sc. and Ph.D. degrees from the University of Maryland, College Park, all in electrical engineering, in 1988, 1990, and 1994, respectively.

He held a postdoctoral appointment in 1994 at the Institute for Systems Research, University of Maryland. In 1995, he joined the Department of Mathematics and Statistics at Queen's University, Kingston, ON, Canada, where he is currently a Professor of mathematics and engineering. Since 1997, he has also been cross-appointed with the Department of Electrical and Computer Engineering at the same university. His research interests include information theory, joint source-channel coding, error control coding, data compression, and digital communications.

Dr. Alajaji currently serves as Editor for Source and Source-Channel Coding for the IEEE TRANSACTIONS ON COMMUNICATIONS. He served as co-chair of the Technical Program Committee of the 2004 Biennal Symposium on Communications and as co-chair of the 1999 Canadian Workshop on Information Theory. In 2001, he received the Premier's Research Excellence Award (PREA) from the Province of Ontario.

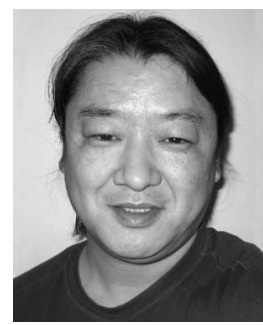

Glen Takahara (M'96) received the B.A. degree in mathematics from the University of British Columbia, Vancouver, BC, Canada, in 1988 and the M.Sc. and Ph.D. degrees in statistics from Carnegie Merlon University, Pittsburgh, PA, in 1990 and 1994, respectively.

Currently, he is an Associate Professor of mathematics and engineering with the Department of Mathematics and Statistics at Queen's University, Kingston, ON, Canada. His research interest are in applied probability modeling in communications networks, sensor networks, wireless ad hoc networks, optical networks, modulation, queuing theory, and statistics.

Dr. Takahara is a member of the IEEE Communications Society. 